\title{
Numerical Analysis of Deformation Behavior of a Water Droplet Impinging on a Solid Surface at Room Temperature
}

\author{
Natsuo HATTA, Hitoshi FUJIMOTO, Hirohiko TAKUDA, Keiko YAMAMOTO, ${ }^{1)}$ Kenji KINOSHITA ${ }^{1}$ \\ and Osamu TAKAHASHI
}

Faculty of Engineering, Kyoto University, Yoshida-honmachi, Sakyo-ku, Kyoto-fu, 606 Japan.

1) Graduate School, Kyoto University, Yoshida-honmachi, Sakyo-ku, Kyoto-fu, 606 Japan.

(Received on February 16, 1995; accepted in final form on April 24, 1995)

\begin{abstract}
In order to predict the deformation process of a liquid droplet impacting on a solid surface at room temperature, the mathematical model has been built up including the effects of surface tension as well as slip mechanism of contact line working in accordance with the degree of wettability on the solid/liquid interface. The numerical droplet deformation process has been compared with the experimental data for some Weber number cases and has been found to be in fairly good agreement not only in the spreading process, but also in the subsequent reducing process. It has been proved that the present model is enough to predict the droplet deformation process. Also, it has been clarified that the droplet deformation process on the solid surface is strongly influenced by the effects of the surface tension as well as the slip phenomenon to keep a proper contact angle.
\end{abstract}

KEY WORDS: droplet deformation process; numerical analysis; free surface; Weber number; surface tension; contact angle.

\section{Introduction}

The cooling technique of a hot surface using water spray is widely used in iron- and steel making industries. The so-called mist or spray cooling is applied to the secondary cooling zone in the continuous casting process. Therefore, many works on the mist/spray cooling were performed from an experimental viewpoint. ${ }^{1-5)}$ However, the useful predictable model of the heat transfer between hot metal surface and water spray has not been established yet, because there are many parameters affecting heat transfer between sprays and hot surface and the effect of these parameters on the cooling mechanism is very complicated. Again, there is a conspicuous lack of knowledge of the structure of mist/spray flows.

From this standpoint, we investigated the two-phase flow in a nozzle as well as in free jet region from numerical and experimental points of view. ${ }^{6-9)}$ Also, the droplet collision process and the subsequent rebounding/disintegrating phenomena after impinging on a hot surface beyond the Leidenfrost temperature were studied from an experimental point of view. ${ }^{10)}$ Furthermore, the numerical analysis of deformation process was performed for the case where a water droplet impacts on a solid surface at room temperature as the first approach. ${ }^{11)}$ The calculated deformation process of a water droplet impacting on the Inconel 625 alloy surface was in good agreement with the measured data only in the spreading process, but an appreciable difference between the two was found in the subsequent process. According to our experimental observation, after a water droplet reaches a maximum diameter, the contact line around a circular wetting area on the Inconel alloy surface moves towards the center. On the other hand, calculated contact line is unmovable, because the no-slip condition is applied to the plate surface boundary.

Practically, we can find some numerical studies treating the droplet behavior after impinging on a plate surface. Harlow and Shannon ${ }^{12)}$ analyzed the droplet deformation process on a surface using the MAC method and compared the numerical result with the experimental ones. However, the effects of viscous and surface tension were neglected in their investigation. Nishikawa et al. ${ }^{13)}$ calculated the deformation process of the droplet of $2 \mathrm{~mm}$ in diameter impinging with the impact velocity of $300 \mathrm{~m} / \mathrm{s}$ on a flat plate by using the SOLA-VOF method. However, we find that the sectional shape of droplet calculated by so doing is far from realistic as the time elapses and no comparison with the experimental data is performed. Turutani et al. ${ }^{14)}$ performed the numerical simulation of the deformation of a droplet impinging on a flat surface with an ordinary room temperature. Their calculation was based upon the SMAC method on the condition that the droplet diameter $\bar{D}_{p}=2 \mathrm{~cm}$. They followed the time history of diameter and height during the deformation on the plate as the characteristic parameters of the droplet deformation and compared the calculated results with the experimental ones. The calculated and experimental results show a similar 
tendency, but are not in agreement with each other. The above mentioned works were restricted to an earlier deformation process. Again, analytical results obtained by accounting for both the surface tension and wettability effects do not seem to have been found yet.

In the present paper, the deformation process of a water droplet impinging on a solid surface at room temperature has been analyzed by taking the surface tension and wettability effects into consideration. The inner flow field of droplet is treated as the axisymmetrical system. In reality, it may be common to consider that when the wettability effect is remarkable, the contact angle of droplet on the surface is small. The converse is also true. Therefore, the fluid at the contact line around the circular wetting area is considered to move in such a way as to maintain a proper contact angle. The moving velocity is assumed to be a function of contact angle alone. The numerical results so obtained are compared with the experimental data to judge the validity of the analytical model and discussed from various points of view.

\section{Basic Equation}

The inner flow field of a droplet impinging on a solid surface obeys the system of axisymmetrical NavierStokes equations for incompressible viscous fluid flow. The following non-dimensional quantities,

$$
\left.\begin{array}{l}
t=\bar{t} /\left(\bar{D}_{p} / \bar{u}_{0}\right), \quad x=\bar{x} / \bar{D}_{p}, \quad y=\bar{y} / \bar{D}_{p} \\
u=\bar{u} / \bar{u}_{0}, \quad v=\bar{v} / \bar{u}_{0}, \quad p=\bar{p} /\left(\bar{\rho} \bar{u}_{0}^{2}\right)
\end{array}\right\}
$$

are introduced into the system of equations. Here, $t$ is the time; $(x, y)$ denote the coordinates in the radial and axial directions, respectively; $(u, v)$ denote the velocity components in the $x$ - and $y$-directions, respectively; $p$ is the pressure. $\bar{D}_{p}, \bar{u}_{0}$ and $\bar{\rho}$ denote the initial droplet diameter, the impinging velocity and the material density of droplet, respectively. Here, overbars denote the dimensional quantities.

The system of equations governing the droplet deformation is composed of the continuity equation and the momentum equations in the $x$ - and $y$-directions as follows:

$$
\begin{gathered}
\frac{\partial u}{\partial x}+\frac{\partial v}{\partial y}+\frac{u}{x}=0 \quad \ldots \ldots \ldots \ldots \ldots \ldots \ldots \ldots \ldots \ldots \ldots \ldots \ldots \ldots \\
\frac{\partial u}{\partial t}+\frac{\partial u^{2}}{\partial x}+\frac{\partial u v}{\partial y}+\frac{u^{2}}{x}=-\frac{\partial p}{\partial x} \\
+\frac{1}{\operatorname{Re}}\left[\frac{\partial^{2} u}{\partial x^{2}}+\frac{\partial^{2} u}{\partial y^{2}}+\frac{\partial}{\partial x}\left(\frac{u}{x}\right)\right] \ldots \\
\frac{\partial v}{\partial t}+\frac{\partial u v}{\partial x}+\frac{\partial v^{2}}{\partial y}+\frac{u v}{x}=-\frac{\partial p}{\partial y}-\frac{1}{F r^{2}} \\
\quad+\frac{1}{\operatorname{Re}}\left[\frac{\partial^{2} v}{\partial x^{2}}+\frac{\partial^{2} v}{\partial y^{2}}+\frac{1}{x} \frac{\partial v}{\partial x}\right] \ldots \ldots
\end{gathered}
$$

in which $R e$ and $F r$ are the Reynolds number and the Froude number, respectively, defined as:

$$
\operatorname{Re}=\bar{u}_{0} \cdot \bar{D}_{p} / \bar{v}, \quad F r=\bar{u}_{0} / \sqrt{\bar{g} \cdot \bar{D}_{p}}
$$

Here, $\bar{v}$ and $\bar{g}$ denote the kinematic viscosity and the gravitational acceleration, respectively.

The pressure balance along the liquid/air boundary (free surface) is given by

$$
p-p_{\infty}=\frac{1}{W e}\left(\frac{1}{R_{1}}+\frac{1}{R_{2}}\right)
$$

where $p_{\infty}$ is the atmospheric pressure, $R_{1}$ and $R_{2}$ are the principal radii of curvature at a given point on the free surface, respectively. $W e$ is the Weber number defined by

$$
W e=\frac{\bar{\rho} \bar{u}_{0}^{2} \bar{D}_{p}}{\bar{\sigma}}
$$

in which $\bar{\sigma}$ denotes the surface tension.

\section{Numerical Procedure}

The flow field has been solved numerically by the finite difference approximation of the Navier-Stokes equations governing axially symmetric incompressible fluid flow. The staggered mesh system has been adopted in the numerical simulation. The second-order upwind scheme, which is commonly called the Donor cell method, has been applied to the convection terms and the second-order central difference scheme has been done to the viscous terms. The droplet deformation process has been analyzed by the MAC type solution method developed by Miyata ${ }^{15)}$ so that the time history of the free surface may be pursued.

The computation of the flow field has been started at the moment when the spherical water droplet having no velocity distribution impinges vertically on the horizontally fixed solid surface $(t=0)$. The physical parameters adopted here are listed in Table 1. The mesh size has been selected to $\Delta x(=\Delta y)=1 / 128$. This corresponds to dividing the initial droplet diameter by 128 . The time interval has been determined by the CFL condition. Here, the CFL number has been selected at 0.1 throughout the present simulation. Also, the no-slip condition $(u=v=0)$ has been applied to the solid/liquid boundary.

In our previous work, ${ }^{11)}$ the calculated deformation process of a water droplet impacting on an Inconel 625 alloy surface at room temperature has been found to be in good agreement with the experimental value only in the spreading process, but the difference between the two has been found to be not necessarily negligible in the subsequent process. According to the experimental observation, the circular wetting area on the surface decreases gradually and reaches a stable state, after the droplet diameter takes a maximum value. On the other hand, the numerical wetting area does not change in the later stage, because the no-slip condition is applied to the liquid/solid interface and thereby the contact line

Table 1. Physical parameters adopted in this study. 
around the circular wetting area is unmovable towards the center owing to the numerical procedure.

Therefore, in the present study, the moving contact line technique has been introduced into the analytical model in such a way as to predict the droplet behavior on the surface more precisely. Concretely, the slip mechanism of the contact line on the solid/liquid interface has been contrived to work, when the velocity field of droplet inside is regarded as being nearly stagnant. It is assumed that the slip velocity is given by a linear function of the contact angle according to Patrick and Michael $^{16)}$ as follows:

$$
u_{\mathrm{slip}}=\bar{a}(\bar{\Theta}-\bar{\theta})
$$

where $u_{\text {slip }}$ is the slip velocity of the contact line, $\bar{\Theta}$ and $\bar{\theta}$ denote a characteristic contact angle between the solid surface and the free surface in the final (steady) state and the temporary contact angle, respectively. The constant value $\bar{a}$ has been determined by the trial and error method and $\bar{a}=-4.85 \times 10^{-5}(1 / \mathrm{rad})$ has been selected throughout the present simulation. It should bear in mind that the value of $\bar{a}$ is applicable to only the case of water/Inconel interface. This procedure has been applied when the velocity components at the computational point adjacent to the contact line along the free surface are nearly zero. The reason why the slip velocity condition is applicable only in the later process of the deformation, is because the slip velocity occurring owing to the wettability on the solid/liquid interface is assumed to be enough small in comparison with the moving rate of the contact line in the spreading process in the present situation.

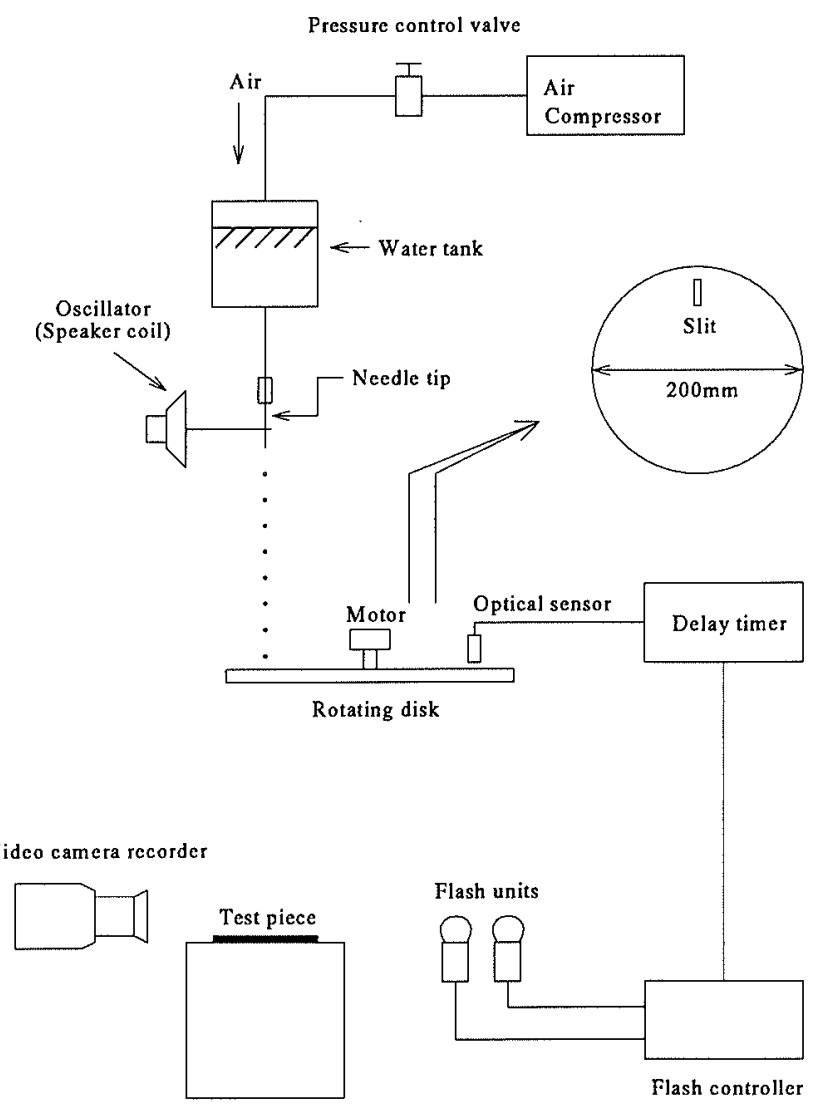

Fig. 1. A schematic diagram of experimental apparatus.

\section{Experimental Apparatus}

A schematic diagram of the experimental apparatus is shown in Fig. 1. It consists of the needle tip to form and release water droplets, the rotating disk to pass through only one droplet from a series of falling droplets, the test plate (Inconel 625 alloy), two micro flash units, the delay timer and the video camera recorder to observe the droplet deformation process. The time histories of the droplet deformation process on the surface have been followed by a number of double exposure video images on the basis of the statistical procedure. The time history of droplet height and width are measured in this study. Also, the measurement accuracy of the droplet size is within $\pm 6 \mu \mathrm{m}$. At any rate, details of the apparatus and of the measurement method are described elsewhere. ${ }^{10,11)}$

\section{Results and Discussion}

First, the validity of mathematical model adopted in this simulation has been examined by comparing the numerical results with experimental ones. Figure 2 shows the comparison of the calculated time history of the deformation process of a droplet impinging on a solid surface (Inconel 625 alloy) with the experimental data for some cases where $(W e, R e)=(22.8,865)$ (a), $(W e, R e)=(27.4,878)(\mathrm{b}),(W e, R e)=(35.8,1054)(\mathrm{c})$ and $(W e, R e)=(47.1,1256)(\mathrm{d})$, respectively. In these figures, the solid lines denote the calculated results and the empty and solid circles are the experimental droplet height and width, respectively. Also, the dotted line shows the time $t_{s}$ when the slip mechanism begins to work. By the way, the definition of the droplet height $H$ and width $W$ is shown in Fig. 3. Here, $t=0$ is the moment of impacting of droplet on the surface. It may be reasonable to consider that the calculated results are in fairly good agreement with the experimental values for all cases. Therefore, we believe that the mathematical model proposed in the present study is a tool which can analyze the deformation process of droplet impinging on the surface.

Now, the collision dynamics of the droplet with the solid surface are analyzed from numerical and experimental points of view. The droplet height $H$ decreases in proportion to the impinging velocity in an early stage. Subsequently, the measured droplet height $H$ keeps almost constant and increases abruptly, although it is not so remarkable, at $t \simeq 2.4$ (a), 2.6 (b), 3.0 (c) and 3.7 (d), respectively. This phenomenon occurs in an earlier time for the lower Weber number case. The abrupt increase in height may occur due to the fact that the rising flow is formed at the center, as will be mentioned later. But, in the calculated results, such phenomena do not appear so conspicuously as the measuring data. The droplet width $W$ increases rapidly in an early stage. Thereafter, $W$ reaches a maximum diameter. It is understood that the maximum diameter tends to increase for the higher Weber number case. In our previous experimental work, ${ }^{10)}$ it was found that the maximum diameter of droplet impinging on a hot surface above 

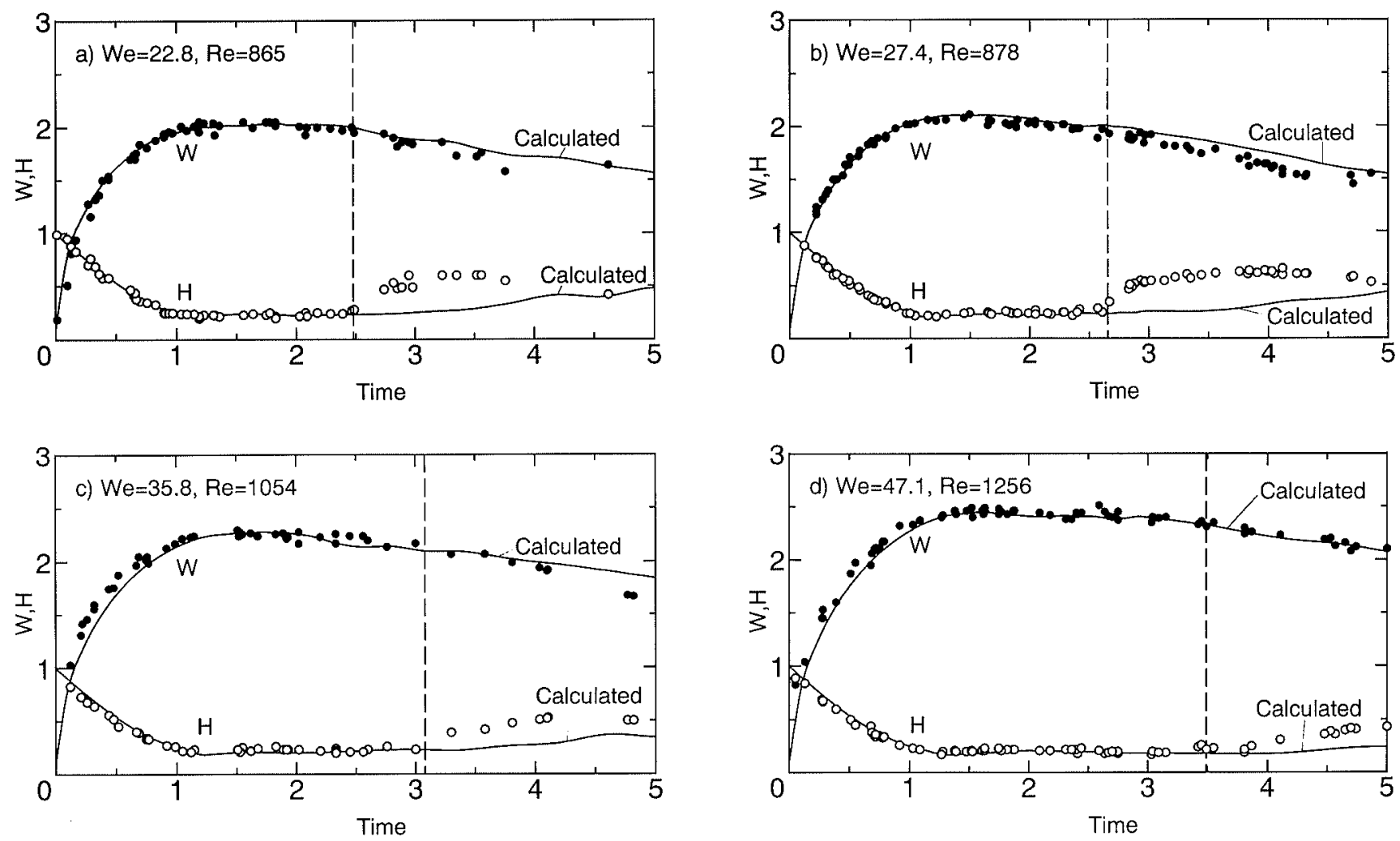

Fig. 2. Comparison of numerical time history of droplet height $H$ and width $W$ with experimental ones for $(W e, R e)=(22.8,865)(a),(27.4,878)(b),(35.8,1054)$ (c) and $(47.1,1256)$ (d), respectively. Note that $H, W$ are non-dimensionalized by the initial spherical droplet diameter $\bar{D}_{p}$ and $t$ is done by $\bar{D}_{p} / \bar{u}_{0}$.
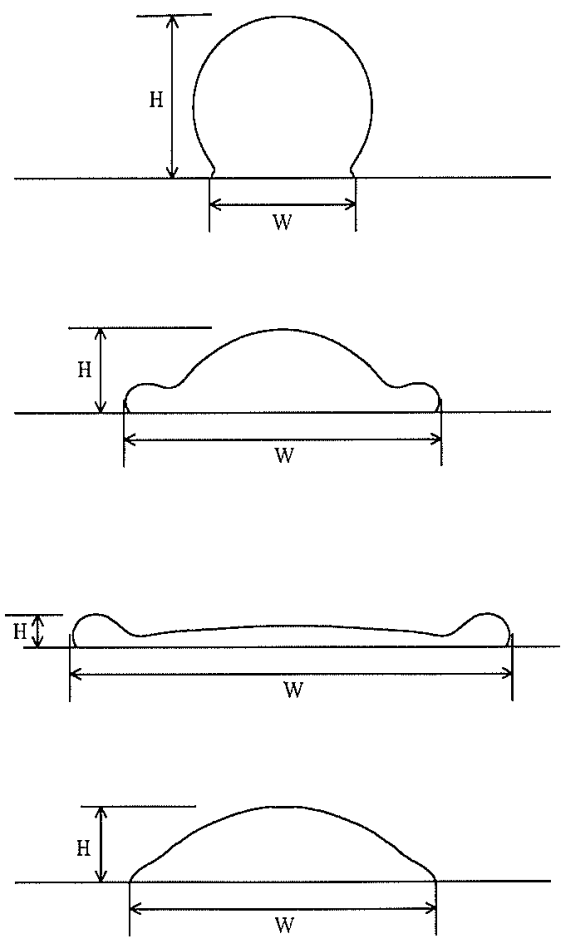

Fig. 3. Definition of non-dimensional droplet height $H(=$ $\left.\bar{H} / \bar{D}_{p}\right)$ and width $W\left(=\bar{W} / \bar{D}_{p}\right)$.

the Leidenfrost temperature is given by a function of the Weber number alone as follows:

$$
D_{p \max }=0.093 W e^{0.74}+1
$$

in which this formula holds true in the range of $W e<50$.
According to this formula, the maximum diameter on the hot surface can be estimated to be $D_{p \max }=1.94$ for $W e=22.8$ (a), $D_{p \max }=2.08$ for $W e=27.4$ (b), $D_{p \max }=$ 2.31 for $W e=35.8$ (c), $D_{p \max }=2.61$ for $W e=47.1$ (d), respectively. Also, according to the present experiment at room temperature, $D_{p \max }$ reaches about 2.0 (a), 2.1 (b), 2.3 (c) and 2.5 (d), respectively. These are somewhat close to the above values, respectively. It may be suggested from these facts that the wetting phenomena occur even on a hot surface almost until the droplet is reaching a maximum diameter. This may be due to a characteristic wettability of the liquid/solid interface. After reaching the maximum width, the droplet width $W$ becomes small gradually in accordance with the degree of the affinity on the water/Inconel alloy interface.

The time when the slip mechanism adopted here begins to work, is $t_{s}=2.48$ (a), 2.65 (b), 3.08 (c) and 3.49 (d), respectively. This time is longer for the higher Weber number case, because it requires long time to lose the kinematic energy owing to the viscous effect and the friction effect on the solid surface.

Figure 4 demonstrates the calculated time history of the free surface configuration and the velocity vector field of droplet inside on the same condition as the case of Fig. 2(d). It is noted that the magnitude of the velocity vector in this figure is comparable at every time stage. The water film radially expands in an early period after impacting of the droplet on the plate surface $(t<1.2)$. Thereafter, the droplet diameter takes a maximum value at $t \simeq 1.6$ (see also Fig. 2(d)). In the peripheral roundish region, a ring vortex structure is observed $(t=1.8)$. Subsequently, the reversal flow from the periphery to 

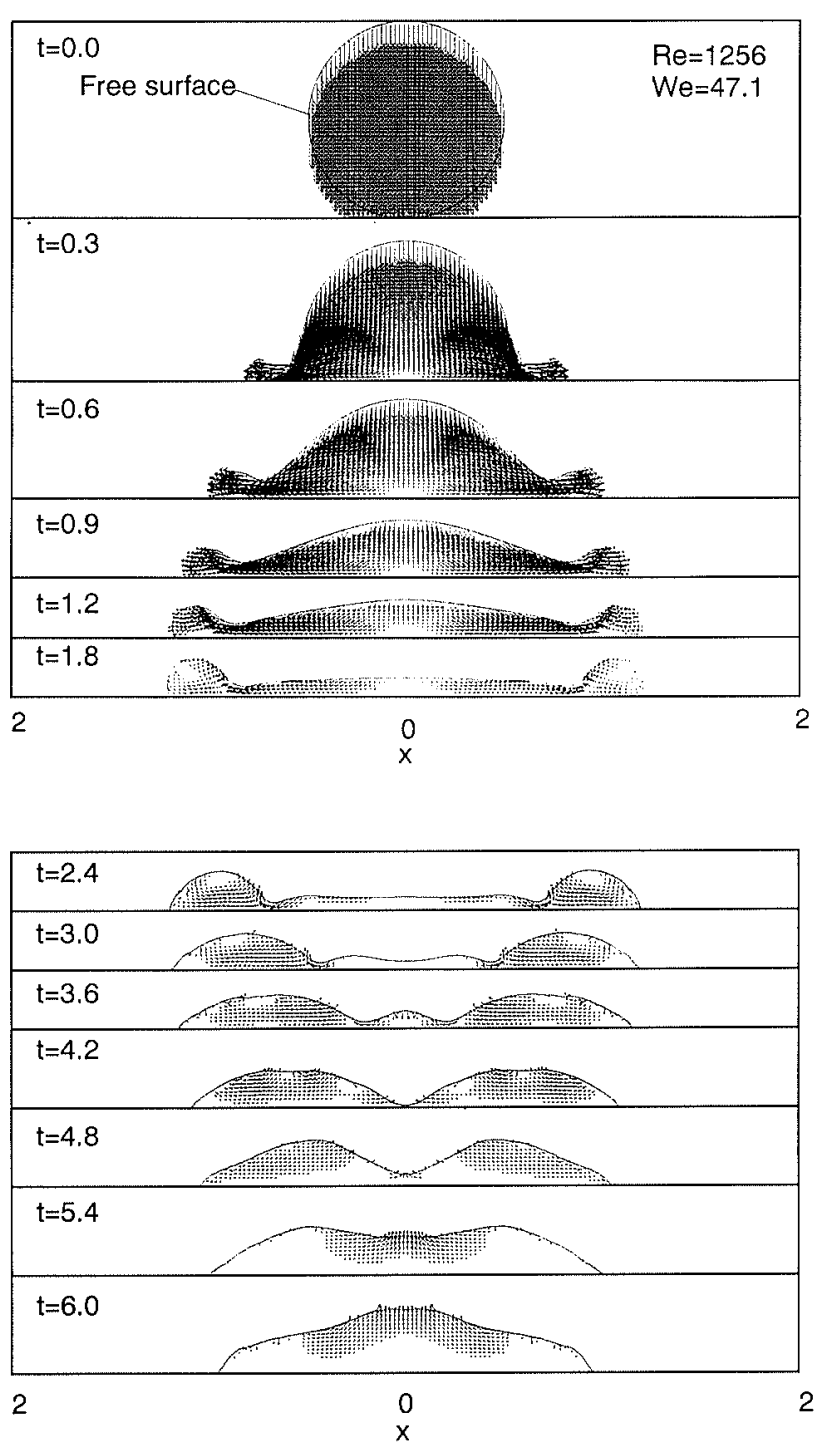

Fig. 4. Numerical free surface configuration and velocity vector field of a droplet impinging on a surface for $W e=47.1$ and $R e=1256$.

the center occurs owing to the surface tension effect $(t>1.8)^{11)}$ and comes into collision with the flow from the center towards the periphery. The collision of the two flows leads to a rising flow and thereby the round edge zone grows in both thickness and width. Furthermore, the flow towards the center overcomes the opposite flow and the position where the two flows collide with each other moves towards the center with time. Thus, the central part of the water film is elevated again.

The temporary contact angle between the free surface and the solid surface is larger than $\pi / 2(\mathrm{rad})$ in the spreading process $(t<1.8)$. In the subsequent process, the temporary contact angle decreases owing to the migration of the volume from the periphery to the center and becomes smaller than the characteristic contact angle on the water/Inconel alloy interface. After $t_{s}=$ 3.49 , the difference between the temporary and characteristic contact angles leads to the slip of the contact line towards the center. As a result, the droplet width $W$ reduces as time elapses.

To tell the truth, it is difficult to keep the droplet

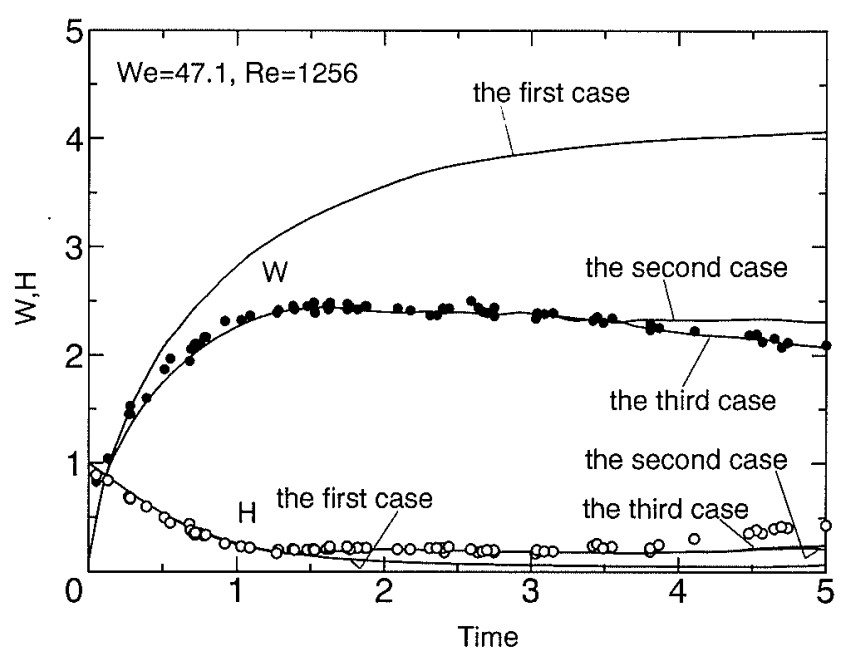

Fig. 5. Time history of droplet height and width for $W e=47.1$ and $R e=1256$. The first case where both the surface tension and slip mechanism are neglected, the second case where only the surface tension is taken into account, and the third case where both the surface tension and slip mechanism are accounted for, have been examined.

volume precisely constant in the present mathematical model, because it is almost impossible to adjust the contact angle in the constant volume state. In the case of Fig. 4, the rate of the droplet volume at $t=6.0$ to the initial one is 0.965 and thereafter decreases monotonically with time. The rate reaches 0.876 at $t=10$. In general, it is difficult to maintain the volume conservation when the flow with free surface is treated even by other numerical analysis such as the VOF (Volume of Fluid) method $^{17)}$ and WC (Weak Constraint) method, ${ }^{18)}$ etc.

Finally, the effects of surface tension as well as the adjustment of contact angle on the droplet deformation process have been investigated. The first case where both the surface tension and slip velocity effects are neglected, the second case where only the surface tension effect is taken into account, and the third case where both the surface tension and the adjustment of contact angle are accounted for, have been compared and examined. Figure 5 shows the comparison between the experimental and calculated time histories of the droplet height and width. The calculation has been performed on condition that $W e=47.1$ and $R e=1256$ corresponding to Fig. 2(d) for the above mentioned three cases. The droplet height shows the same tendency in an early stage regardless of whether surface tension is taken into consideration or not. On the other hand, the difference of the droplet width is much remarkable between the first and second (and third) cases. Then, it has been confirmed that the surface tension has an appreciable influence on the droplet deformation in the width (radial) direction, but no significant influence on the deformation in the height (axial) direction. Next, comparing the second case with the third case, there seems to be an appreciable difference in the droplet deformation in the radial direction after $t_{s}=3.49$, because the slip mechanism is applied to the adjustment of contact line after that time, as mentioned above. It is clear that the calculated time history of the deformation process in the radial direction for the third 
case agrees very well with the experimental data, although the time histories of the droplet height are almost similar between the second and third cases. Therefore, it may be judged that the slip mechanism adopted in the present numerical model makes an important role in order to predict the high accuracy flow field.

\section{Conclusion}

The deformation process of a water droplet impinging on a solid surface at room temperature has been analyzed from numerical and experimental points of view. The numerical model adopted in this study includes the effects of the surface tension as well as slip mechanism due to the affinity corresponding to the degree of wettability on the solid/liquid interface. The effect of the affinity on the solid/liquid interface has been modeled by introducing the slip mechanism of the contact line around the circular wetting area on the surface. The numerical results have been compared with the experimental values and are in fairly good agreement not only in the spreading process, but also in the subsequent reducing process. Also, it is found that the experimental maximum diameter of a droplet on the surface at room temperature for $W e<50$ is very close to the case of the hot surface above the Leidenfrost temperature. Next, the flow field of the droplet inside and the surface configuration of droplet have been investigated by the numerical simulations. The spherical droplet deforms a discoidal shape on the surface and subsequently transforms a lens-shaped mass. Finally, the effects of the surface tension and the slip mechanism on the deformation process have been demonstrated by numerical simulations and it has been concluded that the droplet deformation process on the solid surface is remarkably influenced by the effects of the surface tension as well as the slip phenomenon to keep a proper contact angle.

\section{Acknowledgement}

The authors note that this study has been supported through the Grant in Aid for the Scientific Research (07455287) of the Ministry of Education, Science and Culture in Japan and the Kawakami Memorial Foundation.

\section{Nomenclature}

$\bar{a}: \quad$ slip velocity constant (see Eq. (8))
$\bar{D}_{p}:$ initial droplet diameter

$\bar{D}_{p \max }$ : maximum spreading diameter of a droplet on a surface

Fr: Froude number

$p:$ pressure

$p_{s}$ : atmospheric pressure

$R_{1}, R_{2}$ : principal radii of curvature along free surface

Re: Reynolds number

$t:$ time

$t_{s}$ : time when slip velocity effect is introduced

$u, v$ : velocity components in $x$ - and $y$-directions, respectively

$u_{\text {slip }}:$ slip velocity of contact line

$\bar{u}_{0}$ : impinging velocity

We: Weber number

$x, y:$ coordinates

$\bar{\rho}:$ density of fluid

$\bar{\sigma}:$ surface tension

$\bar{\Theta}$ : characteristic contact angle

$\bar{\theta}$ : temporary contact angle during droplet deformation

\section{REFERENCES}

1) M. Mitsutsuka and K. Fukuda: Tetsu-to-Hagané, 65 (1979), 608.

2) M. Mitsutsuka and K. Fukuda: Tetsu-to-Hagané, 69 (1983), 262.

3) C. Köhler, R. Jeschar, R. Scholz, J. Slowik and G. Borcardt: Steel Res., 61 (1990), 295.

4) J. Slowik, G. Borcardt, C. Köhler, R. Scholz and R. Jeschar: Steel Res., 61 (1990), 302.

5) H. Okubo and S. Nishio: Tetsu-to-Hagané, 79 (1993), 497.

6) N. Hatta, R. Ishii, H. Takuda, K. Ueda and J. Kokado: Trans. Iron Steel Inst. Jpn., 28 (1988), 930.

7) N. Hatta, H. Fujimoto, R. Ishii and J. Kokado: ISIJ Int., 31 (1991), 53.

8) N. Hatta, R. Ishii and H. Fujimoto: Trans. ASME J. Fluids Engg., 114 (1992), 420.

9) N. Hatta, H. Fujimoto and H. Takuda: Appl. Sci. Res., 50 (1993), 129.

10) N. Hatta, H. Fujimoto, H. Takuda, K. Kinoshita and O. Takahashi: ISIJ Int., 35 (1995), 50.

11) N. Hatta, H. Fujimoto and H. Takuda: To be published in Trans ASME J. Fluids Engg. in September, 1995.

12) F. H. Harlow and J. P. Shannon: J. Appl. Phys, 38 (1967), 3855

13) N. Nishikawa, S. Amazu and T. Suzuki: J. Soc. Simul. Tech., 7 (1989), 27.

14) K. Turutani, M. Yao, J. Senda and H. Fujimoto: JSME-B, 55 (1989), 814.

15) H. Miyata: J. Compu. Physics, 65 (1986), 179.

16) J. H. Patrick and J. M. Michael: J. Fluid Mech., 223 (1991), 57.

17) B. D. Nichols, C. W. Hirt and R. S. Hotchkiss: Los Alamos Sci. Labo. Report LA8355, (1980).

18) G. Ryskin and L. G. Leal: J. Comput. Phys., 50 (1983), 71. 